\title{
Study on the Current Evaluation and Indicator System of Network Information Resources
}

\author{
Hai-Yan ZHAO ${ }^{1, a,{ }^{*},}$, Yi HE ${ }^{1, b}$, Xiang-Yang LIU ${ }^{1, c}$, Yu-Yang DU ${ }^{1, c}$ and \\ Xiao ZHANG ${ }^{1, c}$ \\ ${ }^{1}$ Xi'an Communication Institute, Xi'an 710106, China \\ a51375908@qq.com, ${ }^{\text {b8 } 813086903 @ q q . c o m, ~}{ }^{5} 583822272 @ q q . c o m$ \\ ${ }^{*}$ Corresponding author
}

Keywords: Network Information Resources, Evaluation, Indicator.

\begin{abstract}
In this article, we refer to literature and review the function of network information resources and conclude the current evaluation of network information resources. Based on these works, we developed a three-layer network information resources with 25 evaluation indicators of 4 parts and did detailed explanation of each indicator respectively.
\end{abstract}

\section{Introduction}

Internet information resources are exploding, so people find it more difficult to search useful information. There are good and bad Internet information resources, which are hard for us to determine the authenticity of the information space disorder and the overall quality of the phenomenon of decline[1]. Many of the valuable information is submerged into a large variety of redundant information. In order to facilitate the majority of Internet users using Internet information resource information, we need to evaluate Internet information resources, which plays an important role in navigation and guidance of the network of information resources in the network user information during the process of Internet information resources.

\section{Features of Network Information Resources}

Large Amount. Internet is a communication network based on TCP "P protocol, uniting millions of computer networks in different countries and different organizations. It is an extremely huge information resources network. Since everyone can ads on the Internet, it has become a huge information sources. So far, there are thousands of database and numerous other information. Because of the rate features of copying the computer information, the increasing rate of these resources is not comparable[2].

Open Resources. The transfer and exchange of network information resources is totally free from the constraints of space and time, achieving a share of the whole society. Any units and individuals can publish on the Internet anytime, anywhere access to information. Thus, on the one hand the exchange of information becomes smooth and convenient; on the other hand, information flooding and pollution is getting worse[3].

Abundant Content. Almost all-encompassing network of information resources covers different disciplines, different areas, different regions, information resources in different languages. It contains both text and images, sounds and other media information.

\section{Current Evaluation of Internet Information Resources}

Incomplete Indicator System. From the existing indexes or standard, it failed to fully reflect the characteristics of the network information resources inside and outside. People cannot use any of 
them to evaluate the system of a comprehensive, objective, systematic network of information resources. Listed in various standard of system, none of those can be used in practice, which means the none of the existing system can both evaluate qualitatively and quantitatively. Most of them can only be used for the evaluated factors for network information resources. In addition, in the existing indicator system, qualitative indicators are the core, which lacks of necessary quantitative indicators. It is difficult to fully reflect the characteristics of network information resources, so that the evaluation results are hard to be accurate and objective.

Blur Objects of Indicators. From the existing indicators or standards, some specifications apply to the evaluation of the site or page which does not apply to specific content on the site, and some apply to specific content on the site, which does not apply to the site or pages evaluation. Some metrics are useful for evaluating academic sites, some suitable for evaluation of commercial websites; and some single article document applies to the evaluation, but some groups for evaluation of network information resources. However, scholars did not distinguish between levels of indicators, but only for network information resources macroscopic evaluation. Thus, existing indicators of suitable vague did not apply to the evaluation website or index web page in order to distinguish specific information or instructions.

Not Reasonable Design of Indicators. Some indicators literal expression are different, but the meanings are the same or similar, such as "Credibility" and "Accuracy" and so on. In addition, some expressions are too obscure, which are difficult to understand, such as "CriticalThinking". Indicator design is not reasonable, existing the repeated and obscure phenomenon.

Unclear Description to Indicators. In terms of the meaning of the indicators, there are three problems: lacking of detailed explanations to indicators; due to the different understandings, people tend to have different explanations to indicators. Such as "comprehensiveness" explanation, some interpreted as "information resources to fully express the characteristics of a theme point of view", some interpreted as "Coverage comprehensive range"; Interpretation is not accurate of meanings, such as "usefulness" is explained as "in line with the actual needs of the library." The three kinds of situation will bring misunderstanding of network users, influencing the judgment of the quality of network information resources qualities.

Bad Practical Operation of Indicators. Some indicators may seem reasonable, but the specific use of the measure and have considerable difficulty, not practical. Such as: "fairness" and "world view." It can be said either research or practice has tended to focus on qualitative analysis, lacking of quantitative methods, indicators of level indicators of the type, as well as in-depth discussion and the study how the lack of indicators and other basic theory assignment.

\section{Development of the Network Information Resources Evaluation Indicator System}

According to the statistics and analysis of different research institution and scientists to the evaluation indicators of network information resources, we can obtain the evaluation indicators in accordance with the basic principles of network resources information (Table 1).

\section{Evaluation Indicators of Information Content}

Accuracy. During the evaluation, it is mainly to see whether these data is inconsistencies between facts, whether it is from the authorities or the public release and legal documents to withstand the scrutiny and verification. Articles should clearly show the complete dependence of data and in accordance with the full expression of the accuracy of the information. 
Table 1. Evaluation indicators of network information resources

\begin{tabular}{|c|c|c|}
\hline $\begin{array}{c}\text { 1-level } \\
\text { indicators }\end{array}$ & 2-level indicators & 3-level indicators \\
\hline \multirow{25}{*}{ 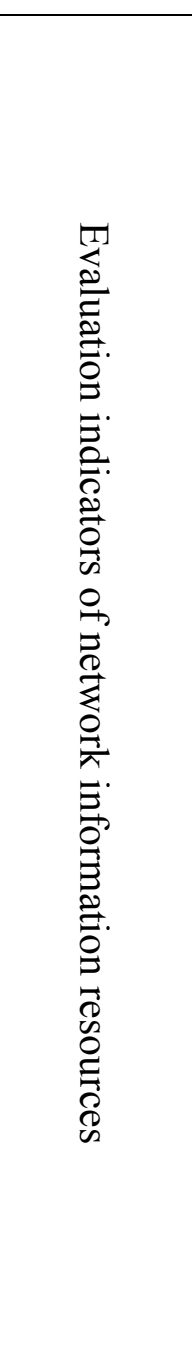 } & \multirow{8}{*}{ Information content } & Accuracy \\
\hline & & Authority \\
\hline & & Extent and depth \\
\hline & & Purpose \\
\hline & & Objectivity \\
\hline & & Originality \\
\hline & & Innovation \\
\hline & & Timeliness \\
\hline & \multirow{6}{*}{ Mnemonic } & Structural Design \\
\hline & & Exterior design \\
\hline & & Multimedia effects \\
\hline & & Compatibility \\
\hline & & Interactive \\
\hline & & Mnemonic \\
\hline & \multirow{7}{*}{$\begin{array}{l}\text { Ease of use } \\
\text { performance }\end{array}$} & Navigation Design \\
\hline & & Friendly interface \\
\hline & & $\begin{array}{l}\text { Transmission speed } \\
\text { and quality }\end{array}$ \\
\hline & & Searchability \\
\hline & & Stability \\
\hline & & $\begin{array}{l}\text { Environmental } \\
\text { requirements }\end{array}$ \\
\hline & & Safety \\
\hline & \multirow{4}{*}{ Others } & Costs \\
\hline & & Citation \\
\hline & & Connections \\
\hline & & Number of visits \\
\hline
\end{tabular}

Authority. It is mainly verified through the author, the website organizers, citing sources, quality control evidence, and several other aspects of the proportion of professional information. For example, the evaluation of information resources for academic networks, including the author's identity and academic status may reflect the quality of information from which it spread to some extent. Quality control evidence is mainly performed in three aspects: from authoritative sites; an editorial review of online journals, usually listed editorial review of processes and methods, editorial board; sources of information quality control process books and journals. There is also a professional information ratio.

Extent and Depth. It looks at whether the scope of its collection is comprehensive and wide-ranging; whether received only national, native language information; what discipline or subject areas covered; included within the scope of subject topic, how wide level, whether there are any omissions; the collection of information is limited within a specific time frame; if it includes both the text message and the image information; if it only to provide the original documents, and provides links to resources; whether relevant bibliographic provides both primary literature, but also provides secondary literature; whether it includes various forms of electronic literature .

Purpose. The main purpose of the study to see when sites, pages are commercial or academic, research is still universal in nature. Generally, academic and higher network level study have higher quality of information. 
Originality. Typically the website that publish the original information are drawn from under scrutiny, compared with those who simply copying others information site. and it has relatively strong originality. It is worth noting that, the original so-called innovation is a difference does not really mean original, but to whether the release of information is the original.

Innovation. Innovative includes two aspects: First, the originality or novelty, covers the topics of information, ideas and opinions and studies the expression of the author's method of application-specific information, which is novel and unique content; the second is provided by the website information in a range of disciplines, forms, methods, etc., or whether there is a unique innovation.

Timeliness. Timeliness is mainly from the creation date of the document, release date, last modification date and other aspects largely determine. It can also be presumed site update frequency, and other relevant information from the copyright validity. A website update frequent information faster, it provides the stronger information on the timeliness, the greater the value to be used.

\section{Layout and Design of Indicators}

Design of Layout. Simply speaking, to enter a website, we need a few mouse clicks to find specific information. The more organizational levels, the larger volume of information. But the user retrieves more convenient.

Exterior Design. Comfortable and beautiful appearance obviously has a strong appeal to users. Beautiful appearance, can make people view the information at the same time enjoy the beauty, relieve fatigue Internet. The preferred site or page should have a beautiful appearance appreciation, but also to guide practically. Exterior design includes the page layout, design of picture and color matching.

Multimedia Effects. Media forms includes two meanings, one of network information resources is the media form; the second is the website that provides information on the forms of media people.

Compatibility. Refers to the network information itself is completely independent, and is relatively independent of the application of computer systems that can be applied to a variety of applications.

Interactive. Immediate, direct interaction is one of the characteristics of the network have. This feature is what printed literature lacks. For example, in many sites and pages, it will be a significant representation of the organization, institution or author's home address, email or contact information, which is convenient for users to quickly and easily communication. Also, if the site opened a interactive form, it can provide a variety of interactive interface for viewers with the author or related personnel immediate and convenient communication. It is clearly that the quality of the site can be greatly improved.

\section{Ease of Use Indicators}

Navigation Design. Issues of navigation design involves: whether internal links are abundant, whether resources to maintain links are novel; whether the linked content can normally access, whether the resource is linked with the themes and subject-related; whether the design can provide only text information; whether the design will help in the understanding of the content and so on.

Friendly Interface. User-friendly interface allows users easily access, indirectly raises the value of online information.

Transmission Speed and Quality 
Speed and quality of transmission directly affects the ease of use of people. If the home page graphic document will cost too much valuable time user, response time per click or button operation also affect the length of the transmission quality reasons.

Search ability. It is mainly related to: the level of performance of the search engine, whether it is to retrieve a single or diversity mode, whether it can help browse to search, and also, whether it is to directly enter search terms to seawrch, whether it can be searched by title, author, keywords, and other ways, whether it is to provide advanced query way of the inquiry information, which is selectively with limited privileges.

Safety. It looks at a website or web security which mainly depends on its anti-virus and the anti-virus capabilities, whether the specific information has a dedicated server, whether the design uses security encryption measures.

\section{Others}

Costs. Costs is represented in two aspects: technical support and connection costs. Technical support mainly refers to the use of the device to find resources environment. Connection costs include communications and information traffic charges.

Citation. Generally, the higher the number cited and explained the situation which is incorporated by reference, the more trustworthy of the academic resources.

Link Situations. First, it is a link from the site (pages) to other sites (pages); the other is a link from another site (page) to the site (page).

Number of Visits. Under normal circumstances, the more often browsed site or page is visited more often and more reliable.

\section{Acknowledgement}

This research was financially supported by The national social science fund(14GJ003-029).

\section{References}

[1] Juha Kettunen, The strategic evaluation of academic libraries, Library Hi Tech . 3(2007) 51-58.

[2] John Carlo Bertot, Libraries and networked information services: issues and consideration in measurement, Performance Measurement and Metrics. 1(2004)32-37.

[3] R N. MaIviya, A Kumar, Networking and Consortia Management Techniques, DESI DoC Bulletin of Information Technology. 3(2007):21-30. 